


\title{
APORTACIONES A LA DISTRIBUCIÓN Y ECOLOGÍA DE LAS CAROFÍCEAS DE LA PROVINCIA DE VALENCIA
}

\author{
José Luis CARRETERO
}

\begin{abstract}
RESUMEN. Aportaciones a la distribución y ecología de las Carofíceas de la provincia de Valencia. Se aporta información sobre la distribución y ecología de las carofíceas de la provincia de Valencia, a partir del estudio de 88 localidades distintas. De los 15 táxones registrados, Chara canescens, $C$. connivens y C. galioides corresponden a nuevas citas provinciales. Se presentan los principales parámetros físicoquímicos del agua para cada especie.
\end{abstract}

Palabras clave. Charophyceae, corología, ecología, Valencia (E de España).

SUMMARY. Contributions to the distribution and ecology of the Charophyceae of the Valencia province. As result of the study of 88 places, data on distribution and ecology of the Charophyceae in the Valencia province are given. Fifteen taxa have been surveyed. Chara canescens, $C$. connivens and $C$. galioides are new provincial records. The main physical-chemical characteristics of water for each species are presented.

Key words. Charophyceae, Chorology, Ecology, Valencia (E Spain).

\section{INTRODUCCIÓN}

Este artículo tiene como objetivo fundamental ampliar la información dada por autores anteriores sobre la distribución y ecología de los carófitos de la provincia de Valencia, tanto la de carácter puntual de Reyes Prósper (1910), Pardo (1942), Corillion (1962), Batalla (1975), Margalef Mir (1981) y Pedrola (1989), como la más extensa de Boira y Carretero (1985). Para ello, se han identificado los táxones encontrados en la mayor parte de los biótopos acuáticos valencianos, recorridos durante el período comprendido entre los meses de agosto de 1990 y 1991, además de recoger muestras de agua para su posterior análisis en el laboratorio.

\section{MATERIAL Y MÉTODOS}

El material vegetal ha sido determinado siguiendo fundamentalmente a Wood \& Imahori (1965), Corillion (1975) y Comelles (1985). En las especies colectivas Chara hispida y $C$. vulgaris, se ha mantenido el criterio de Boira y Carretero (1985).

En las muestras de agua, el $\mathrm{pH}$, la conductividad, la concentración de cloruros, el oxígeno disuelto (OD) y la demanda biológica de oxígeno (DBO) se han analizado de forma instrumental. La alcalinidad se ha calculado potenciométricamente (Rodier, 1981) y la concentración de $\mathrm{Ca}+2+\mathrm{Mg}+2$ según los métodos oficiales del Ministerio de Agricultura (Vallejo, 1986). Los sulfatos, nitratos, 
nitritos, amonio, fosfatos y demanda química de oxígeno (DQO) se han determinado colorimétricamente (Walters, 1989).

\section{Localidades estudiadas:}

Se han realizado un total de 88 inventarios, distribuidos en las zonas de mayor abundancia de biótopos acuáticos (fig. 1). Se han ordenado de acuerdo a valores crecientes de clorinidad y se ha mantenido la clasificación dada por Margalef (1980).

\section{$\mathbf{C l}<\mathbf{0 . 1 0}$ gr/l (Agua dulce)}

1. Higueruelas-Andilla, Las Collalbas, balsa de obra, XK 8209, 2-XI-90.

2. Ayora, balsa de riego de obra, XJ 6725, 5-IV91.

3. Venta Gaeta, charca en barranco, XJ 7553, 5 IV-91.

4. La Yesa, balsa de obra, XK 7815, 8-X-90.

5. Bolbaite, remanso de rambla, YJ 0026, 28-IV91.

6. Sot de Chera, charca en río Reatillo, XJ 7987, 4-V-91.

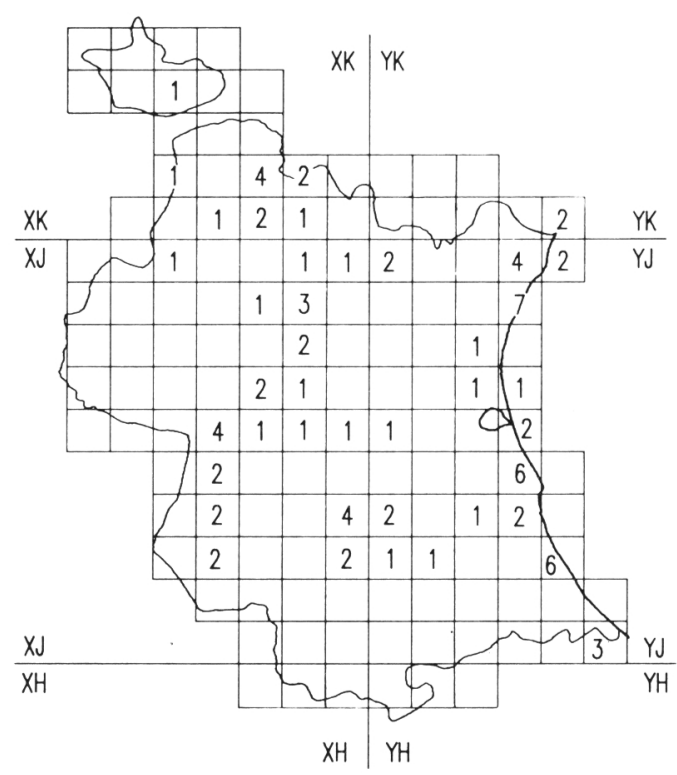

Figura 1. Número de biótopos acuáticos inventariados por cuadrícula de $10 \mathrm{Km}$ de lado.
7. Tuéjar-Utiel, Villanueva, remanso de barranco, XJ 5995, 18-V-91.

8. Eras de Alpuente, remanso de barranco, XK 7018, 17-V-91.

9. Bicorp, balsa de riego, XJ 9133, 9-IfII-91.

10. Eras de Alpuente, remanso de barranco, XK 7018, 28-IX-90.

11. Chiva, fuente La Alóndiga, remanso de arroyo, XJ 8577, 3-VIII-91.

12. Ademuz, Val de la Sabina, remanso de barranco, XK 5135, 20-VIII-91.

13. Alcotas, balsa de riego, XK 7810, 20-VIII-91.

14. Bicorp, riachuelo, XJ 9134, 9-III-91.

15. Chiva, arroyo, XJ 8777, 3-VIII-91.

16. Titaguas, La Caballera, remanso de barranco, XK 5716, 20-VIII-91.

17. Gandía, acequia en marjal, YJ 4320, 5-IX-90.

18. Quesa, charca lateral en río Grande, XJ 9331, 9-III-91.

19. Quesa, Charcas del río Grande, XJ 9228, 28IV-91.

20. Jarafuel, Fuente Bella, balsa del obra, XJ 6536, 29-III-91.

21. Navarrés, La Playa, acequia de obra, XJ 9928 , 20-X-90.

22. Jarafuel, Fuente Bella, remanso de arroyo, XJ 6536, 29-III-91.

23. Macastre, río Magro, XJ 8757, 12-III-91.

24. Gandía, azarbe en marjal, YJ 4321, 25-V-91.

25. Tuéjar, río Tuéjar, XK 6805, 8-X-90.

26. Llombai-Turis, remanso en rambla Algodar, YJ 0255, 12-III-91.

27. Calles-Higueruelas, Fuente Madrid, balsa, XK 7804, 20-VIII-91.

28. La Pobleta, barranco, XK 8512, 2-XI-90.

29. Juan Vich, barranco, XJ 6657, 29-III-91.

30. Juan Vich, charca en fuente, XJ 6857, 29-III91.

31. Gestalgar, La Marjana, remanso de arroyo, XJ 8180, 3-VIII-91.

32. Zarra, río Zarra, XJ 6629, 6-IV-91.

33. Llosa de Ranes, Puerto de Cárcer, charca de fuente, YJ 1224, 6-IV-91.

34. Higueruelas-Calles, Hoya Hantaño, balsa de fuente, XK 7805, 8-X-90.

35. Mijares, charca en río Mijares, XJ 7661, 23-III91.

36. Dos Aguas-Montroy, Fuente del Real, balsa de obra, XJ 9551, 6-IV-91.

37. Gestalgar, remanso en barranco, XJ 8685, 2VIII-91.

38. Juan Vich, balsa, XJ 6757, 29-III-91.

39. Masalavés-Tous, Los Tollos, charca, YJ 0836 , 28-IV-91.

40. Mijares, remanso en río Mijares, XJ 7860, 23 III-91.

41. Yátova, balsa de obra, XJ 8661, 23-III-91. 
42. Liria, charca, YJ 0791, 8-X-91.

43. Bugarra-Gestalgar, balsa de obra, XJ 8987, 3XI-90.

$\mathrm{Cl}$ : 0.10-0.20 gr/l (Agua dulce)

44. Sumacárcer, balsa de riego, YJ 0432, 2-III-91.

45. Casinos, balsa de obra, XJ 9496, 8-X-90.

46. Alcira, rambla, YJ 2136, 20-X-90.

47. Quartell, campo inundado en marjal'litoral, YK 4000, 9-II-91.

48. Favareta-Cullera, arrozal, YJ 3634, 22 -VII-91.

49. Bicorp-Quesa, charca en río Escalona, XJ 9333 , 9-III-91.

50. Cullera, arrozal, YJ 3641, 22-VII-91.

51. La Pobleta, remanso de arroyo, XK 8611, 2-XI90.

52. Cullera-Favareta, arrozal, YJ 3636, 22-VII-91.

53. Sueca-Mareny Blau, arrozal, YJ 3443, 29-VII91.

54. Valencia, Jardines del Real, balsa de obra, YJ 2673, 13-X-90.

55. Cullera-Mareny Blau, arrozal, YJ 3643, 22VII-91.

56. Saltos de Cofrentes, charca en río Cabriel, $\mathrm{XJ}$ 6446, 29-III-91.

57. Las Palmeras-Sueca, arrozal, YJ 3348, 29-VI91.

58. Cullera, arrozal, YJ 3642, 30-VIII-90.

59. El Perelló-Las Palmeras, arrozal, YJ 3549, 29 VI-91.

Cl: 0.20-0.50 gr/l (Agua salobre oligohalina)

60. Xaraco, balsa de riego, YJ 4124, 7-IX-90.

61. El Perelló, arrozal, YJ 3350, 29-VI-91.

62. El Perelló, arrozal, YJ 3451, 29-VI-91.

63. Jalance, charca en río Júcar, XJ 6640, 6-IV-91.

64. Liria, San Vicente, charca, YJ 0892, 8-X-90.

65. El Saler, rastrojo de arrozal inundado, YJ 2961, 29-XII-90.

66. Villar-Bugarra, canal de riego, XJ 8993, 3-XI90.

67. Xaraco, azarbe en marjal, YJ 4025, 22-VII-91.

Cl: 0.50-1 gr/l (Agua salobre oligohalina)

68. El Saler, campo inundado, YJ 3060, 22-I-91.

69. Xaraco, azarbe en marjal, YJ 4125, 22-VII-91.

70. Casas del Río, zanja inundada, XJ 6051, 29-III91.

71. Sagunto, Grao Viejo, rastrojo de arrozal inundado, YJ 3690, 16-III-91.

72. Xaraco, balsa en marjal, YJ 4125, 7-IX-90.

73. Oliva, poceta en marjal, YJ 5507, 19-I-91.

74. Oliva, carrizal inundado, YJ 5507, 19-I-91.

Cl: 1-2 gr/l (Agua salobre oligohalina)

75. Sagunto, Grao Viejo, rastrojo de arrozal inundado, YJ 3690, 5-I-91.
76. Sagunto, Grao Viejo, campo inundado, YJ 3691, 5-I-91.

77. Port Sa Playa-Playa Puebla Farnals, charca, YJ 3281, 9-V-91.

78. Sagunto, La Almardá, zanja en saladar, YJ 3997, 22-IX-90.

79. Sagunto, La Almardá, carrizal inundado, YJ 4000, 9-II-91.

80. Puzol (playa)-Grao Viejo de Sagunto, charca en saladar, YJ 3589, 16-III-91.

Cl-: 2.0-4.2 gr/l (Agua salobre mesohalina)

81. Puzol (playa)-Grao Viejo Sagunto, azarbe en saladar, YJ 3589, 3-IV-91.

82. Puzol (playa)-Grao Viejo Sagunto, rastrojo arrozal inundado, YJ 3589, 5-I-91.

83. Puzol (playa)-Grao Viejo Sagunto, saladar inundado, YJ 3589, 5-I-91.

84. Puzol (playa)-Grao Viejo Sagunto, charca en saladar, YJ 3589, 16-III-91.

85. Oliva, poceta en marjal, YJ 5506, 5-IX-90.

86. Sagunto, La Almardá, charca en saladar, YJ 4099, 16-III-91.

87. Sagunto, La Almardá, campo inundado, YJ 4099, 9-II-91.

88. Puzol (playa)-Grao Viejo Sagunto, saladar inundado, YJ 3589, 5-I-91.

Las localidades con carofíceas que presentan una mayor clorinidad del agua $(0.80$ a $4.20 \mathrm{gr} / 1 \mathrm{de} \mathrm{Cl}^{-}$) son las de las zonas litorales situadas entre Puzol y el límite con la provincia de Castellón. Debido a la ausencia de inundación durante el otoño-primavera de 1990-1991, en las zonas costeras de Puebla de Farnals y El Puig no hemos encontrado carófitos, aunque se hayan registrado en otras ocasiones (Boira y Carretero, 1985). Las aguas salobres oligohalinas son frecuentes en las localidades situadas entre El Saler y el límite con la provincia de Alicante y escasas en todas las zonas interiores de la provincia de Valencia, donde predominan las aguas dulces.

\section{RESULTADOS}

En la Tabla 1 se indican los valores extremos de los principales parámetros ecológicos del agua donde se encuentran los correspondientes táxones. Nitella confervacea (Br̀éb.) 
A. Braun ex Leonh. y N. ornithopoda A. Braun, indicadas por Boira y Carretero (1985) y Pedrola (1989) respectivamente, no han sido encontradas en ninguna de las localidades visitadas en este estudio. En los táxones que no suponen novedad provincial y no se indican antecedentes bibliográficos se amplia la distribución dada por Boira y Carretero (1985); los escasos registros anteriores a estos autores ya se indican en dicha publicación.

Chara aspera Deth. ex Willd.

Localidad: 72.

En balsa de $60 \mathrm{~cm}$ de profundidad, con agua permanente salobre oligohalina. En verano, junto a Chara hispida var. hispida y $C$. vulgaris var. vulgaris. También se ha indicado (Boira y Carretero, 1985) en aguas temporales en primavera.

\section{Chara braunii Gmelin}

Localidades: 48, 50, 57, 59, 61, 62.

Frecuente durante el verano en los arrozales de la Albufera, en aguas someras temporales, desde dulces a ligeramente oligohalinas. Es más abundante en las primeras fases del ciclo del arroz, cuando el cultivo no tienen demasiada cobertura. Generalmente acompaña a Zannichellia peltata Bertol. y/o Chara vulgaris.

\section{Chara canescens Desv. \& Lois.}

Localidades: 80,83 .

En saladar inundado temporalmente durante invierno-primavera, con aguas superficiales fuertemente oligohalinas a ligeramente mesohalinas. Muy escasa, junto a Zannichellia pedunculata Reichenb., Ruppia maritima L. y Chara galioides. Los individuos recolectados corresponden a pies femeninos partenogenéticos. No se tienen referencias de citas anteriores para la provincia de Valencia. Al contrario de los otros registros españoles (Comelles, 1986), nuestras localidades están relacionadas con biótopos litorales.
Chara connivens Salzm. ex A. Braun.

Localidades: 71, 75.

En rastrojo de arrozal inundado temporalmente en invierno-primavera con aguas oligohalinas. Presenta una buena cobertura, junto con Zannichellia pedunculata. Parece ser el primer registro para la provincia de Valencia.

Chara galioides DC.

Localidades: 71, 75, 80, 81, 83, 84, 88.

En marjales inundados temporalmente en invierno-primavera con aguas generalmente someras (a veces en azarbes de $1 \mathrm{~m}$ de profundidad), salobres oligohalinas a mesohalinas. Suele desarrollarse junto a Zannichellia pedunculata, Ruppia maritima, Tolypella hispanica, Chara canescens y/o C. connivens. No se tienen referencias de registros anteriores para la provincia de Valencia.

Chara globularis Thuill.

Localidad: 45.

En balsa de $65 \mathrm{~cm}$ de profundidad con agua permanente dulce, junto a Zannichellia peltata.

\section{Chara hispida L. var. hispida}

Localidades: 18, 19, 21, 22, 35, 40, 44, 67,81 .

En aguas desde dulces a mesohalinas, someras a profundas (hasta $2 \mathrm{~m}$ de profundidad) y en régimen temporal o permanente. Tanto este taxon, como los tres siguientes, se presentan durante todo el año $y$, frecuentemente, forman masas densas prácticamente monoespecíficas.

\section{Chara hispida L. var. major Hartman}

Localidades: 2, 20, 27, 38, 44, 72, 74, 85. En aguas dulces a mesohalinas, someras a profundas (hasta $3 \mathrm{~m}$ de profundidad) y en régimen temporal o permanente.

Chara vulgaris L. var. gymnophylla (A. Braun) Nym. 
Localidades: $33,46,48,50,52,55$.

En aguas dulces, generalmente poco profundas, en régimen temporal o permanente.

\section{Chara vulgaris $\mathrm{L}$. var. vulgaris}

Localidades: 1, 3, 4, 5, 6, 8, 9, 10, 11, 12, $13,14,15,16,17,19,21,23,24,25,26,27,28$, $29,30,31,32,34,36,37,39,40,41,42,43,47$. $49,51,53,54,55,56,58,60,63,64,66,67,70$, $72,73,77,78,82$.

En aguas someras a profundas (hasta $2 \mathrm{~m}$ de profundidad), temporales o permanentes, tranquilas o ligera corriente, dulces a mesohalinas.

\section{Nitella gracilis (Smith) Agardh}

Localidad: 49.

En charca de agua dulce, superficial y en régimen temporal en invierno-primavera, junto a Chara vulgaris var. vulgaris. Ya citada por Pedrola (1989) en Anna (Gongo de la Escalera), en aguas permanentes.

\section{Nitella hyalina (DC.) Agardh}

Localidades: 67, 69.

En azarbes poco profundos con aguas salobres oligohalinas permanentes, junto a Chara vulgaris var. vulgaris.

\section{Nitella tenuissima (Desv.) Kütz.}

Localidades: 18, 56, 70.

En aguas superficiales, temporales (invierno-primavera), de dulces a ligeramente oligohalinas, junto a Zannichellia peltata, Chara vulgaris var. vulgaris o C. hispida var. hispida. Pedrola (1989) la cita en Mogente (embalse El Bosquet) en aguas permanentes.

\section{Tolypella glomerata Desv.}

Localidades: 7, 65, 68, 76, 86.

En aguas poco profundas, temporales (invierno-primavera), desde dulces a mesohalinas. Presenta cobertura variable y crece junto a Chara vulgaris, Tolypella hispanica, Zannichellia peltata o Zannichellia pedunculata.

Tolypella hispanica Nordstedt ex T.F. Allen Localidades: 75, 76, 79, 86, 87, 88.

En aguas superficiales, temporales (invierno-primavera), desde oligohalinas a mesohalinas. Generalmente presenta una buena cobertura y suele formar comunidad con Zannichellia pedunculata.

\section{CONCLUSIONES}

En este estudio se ha ampliado de forma considerable los conocimientos existentes sobre la distribución y ecología de los carófitos de la provincia de Valencia, además de indicar Chara canescens, $C$. connivens y $C$. galioides como novedades provinciales.

Chara vulgaris var. vulgaris, C. vulgaris var. gymnophylla, C. hispida var. hispida y $C$. hispida var. major se distribuyen por toda la provincia (la primera con mucha frecuencia y las otras de forma más esporádica), durante todo el año y en medios con características físico-químicas del agua diversas.

Chara aspera y C. globularis colonizan durante todo el año aguas someras o algo profundas, en régimen estacional o permanente, dulces o oligohalinas, la primera en la marjal de Tavernes de Valldigna a Gandía y la segunda escasamente diseminada por toda la provincia.

Chara braunii sólo se ha encontrado en aguas superficiales, temporales (verano), dulces a ligeramente oligohalinas, de los arrozales valencianos.

Chara canescens, C. connivens, C. galioides y Tolypella hispanica se presentan en aguas someras, temporales, en inviernoprimavera, oligohalinas a mesohalinas, en la zona litoral del NE de la provincia, entre Puzol y el Puerto Viejo de Sagunto las tres primeras (aunque no se han registrado, es posible su presencia al norte del Puerto de Sagunto) y 
\&

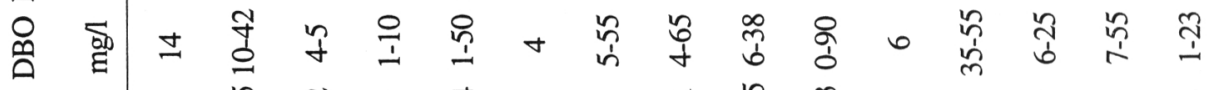

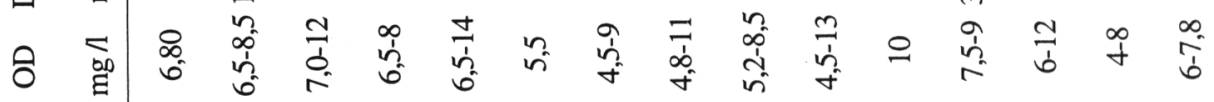

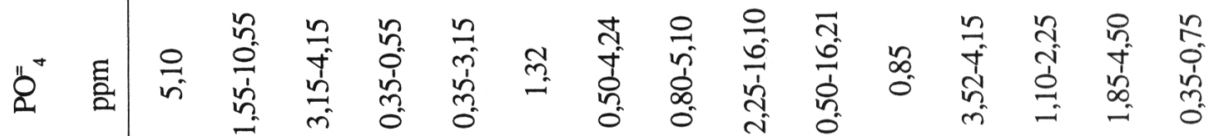

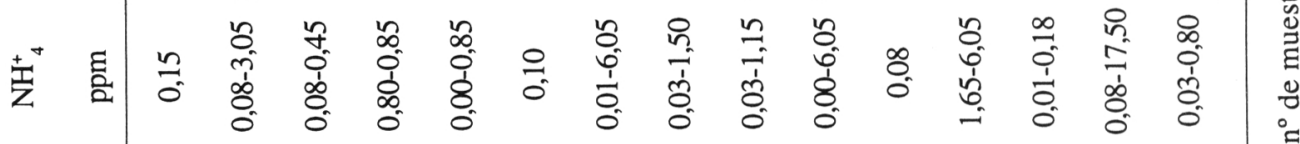

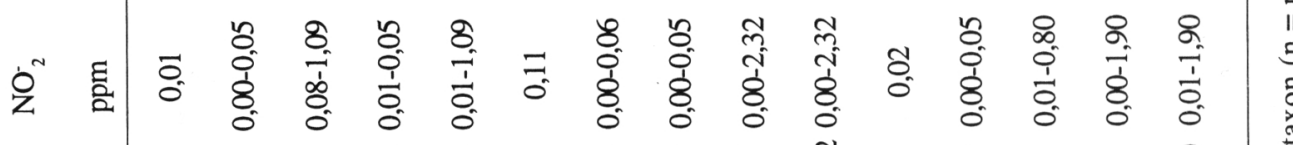

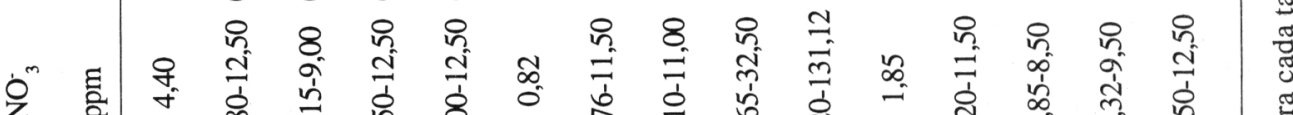

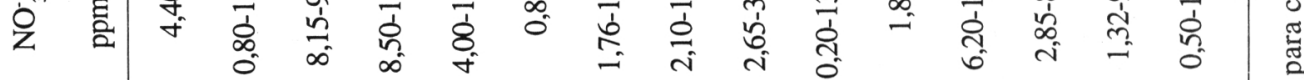

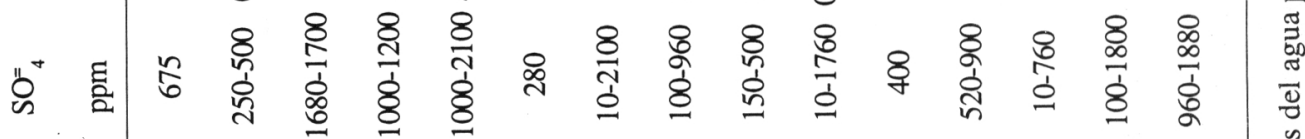

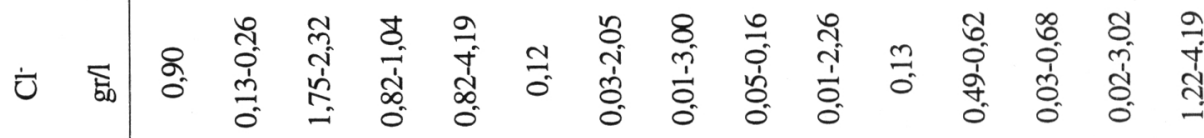

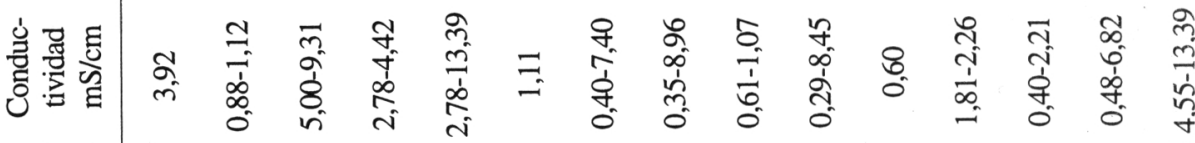

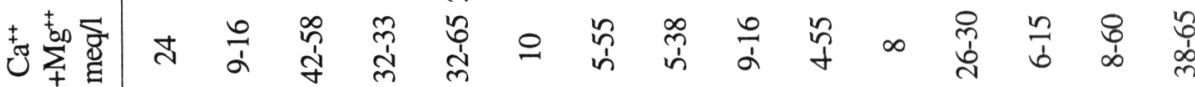

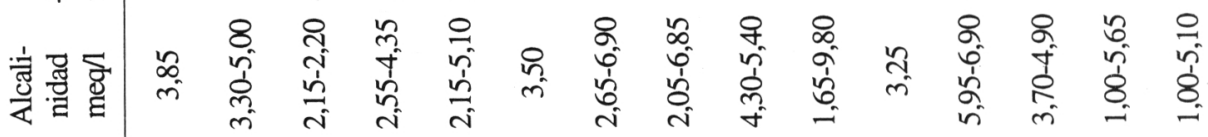

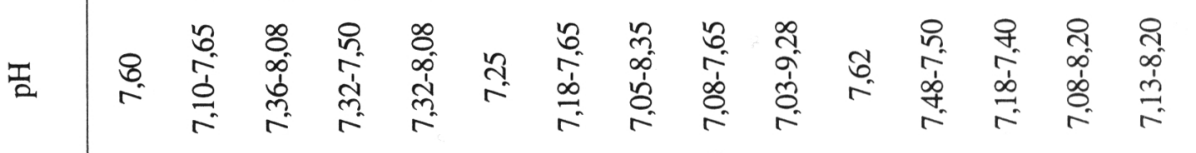

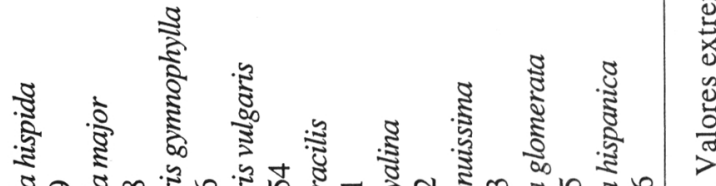

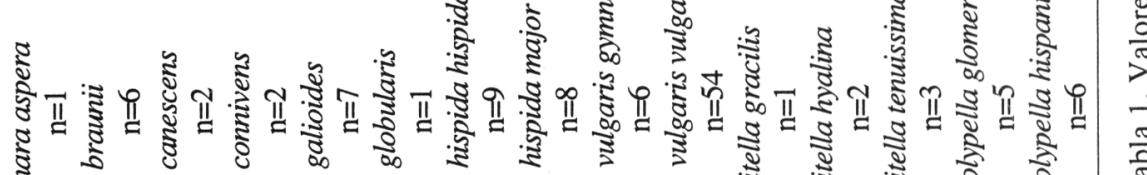

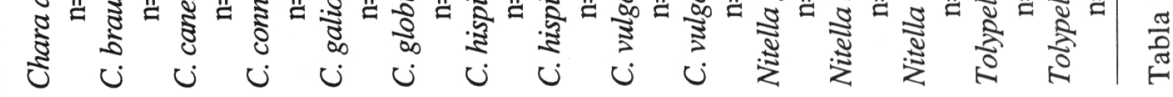


entre Puebla de Farnals y el límite con la provincia de Castellón la última. Las dos primeras son muy escasas, la tercera, medianamente frecuente y la última, muy frecuente.

Tolypella glomerata coloniza durante invierno-primavera aguas estacionales, poco profundas, desde dulces a mesohalinas, de las zonas litorales, especialmente de la zona arrocera de la Albufera y del NE de la provincia, y de algunos puntos interiores.

Nitella hyalina se ha registrado en aguas permanentes oligohalinas de las zonas litorales del sur de la provincia. Nitella confervacea, $N$. gracilis, $N$. ornithopoda y $N$. tenuissima se encuentran escasamente diseminadas, fundamentalmente en invierno-primavera, en aguas generalmente dulces del interior de la provincia.

Todas las carofíceas de la provincia de Valencia se desarrollan en aguas transparentes, tranquilas o con escasa corriente (Chara vulgaris resiste cierta reofilia), con $\mathrm{pH}$ alcalino, en general poco o nada contaminadas (salvo en contadas ocasiones, valores bajos de nitrógeno, fósforo y materia orgánica) y con una proporción de sulfatos variables. El factor ecológico de mayor importancia en la distribución de carófitos (tab. 1) es la clorinidad del agua, siendo la más resistente a la concentración de cloruros Chara galioides, Tolypella hispanica, Tolypella glomerata, Chara hispida, Chara canescens, Charavulgaris y Chara connivens.

\section{BIBLIOGRAFÍA}

BATALLA, J.A. -1975- Las algas de los arrozales y el empleo de los alguicidas. Federación Sindical Agricultores Arroceros de España. Valencia.

BOIRA, H. y J.L. CARRETERO -1985- Las carofíceas de las provincias de Castellón y Valencia. Collect. Bot., 16(1): 13-19.

COMELLES, M. -1985- Clave de identificación de las especies de carófitos de la Península Ibérica. Asoc. Española de Limnología. Barcelona.
COMELLES, M. -1986- Hallazgo de dos poblaciones sexuales de Chara canescens Desv. \& Lois. en España. Anales Jard. Bot. Madrid, 42(2): 285-291.

CORILLION, R. -1962- Nouvelle contribution à l'étude des charophycées de la Péninsule Ibérique et du Maroc Occidental. Bull. Soc. Sci. Bretagne, 37: 65-80.

CORILLION, R. -1975- Flore et végétation du Massif Armoricain IV. Fore des Charophytes du Massif Armoricain et des contrées voisines d'Europe Occidentale. C.N.R.S. Paris.

MARGALEF, R. -1980- Ecología. Ed. Omega. Barcelona.

MARGALEF MIR, R. -1981- Distribución de los macrófitos de las aguas dulces y salobres del $N$ y NE de España y dependencia de la composición química del medio. Fundación J. March. Madrid.

PARDO, L. -1942-La Albufera de Valencia. Inst. Fores. Inv. Exp. Madrid.

PEDROLA, J. -1989- Fragmenta chorologica occidentalia, Algae, 1849-1851. Anales Jard. Bot. Madrid, 45(2): 521.

REYES PRÓSPER, F. -1910- Las Carófitas de España. Imp. Artística Española. Madrid.

RODIER, J. -1981- Análisis de las aguas. Ed. Omega. Barcelona.

VALLEJO, J.M. (ed.) -1986- Métodos oficiales de análisis, III. Suelos y Aguas. M.A.P.A. Madrid.

WALTERS, G.L. (ed.) -1989- Water analysis Handbook. Hach. Co. Loveland, USA.

WOOD, R.D. \& K. IMAHORI-1964/65-A Revision of the Characeae. 2 vols. Verlag von J. Cramer. Weinheim.

Aceptado para su publicación en Julio de 1993

Dirección del autor. Departamento de Biología Vegetal (Botánica). E.T.S. Ingenieros Agrónomos. Universidad Politécnica. 46020 Valencia. 